\title{
Bilateral renal sinus myelolipomas
}

\author{
Ashley Cox, MD; ${ }^{*}$ Saul L. Offman, MD; Jennifer L.O. Merrimen, MD; ${ }^{\dagger}$ Andrea Kew, MD; ${ }^{;}$ \\ Richard W. Norman, MD, FRCSC ${ }^{*}$
}

\begin{abstract}
Adrenal myelolipomas are benign neoplasms consisting of hematopoietic cellular elements and adipose tissue. They are uncommon, found in $0.4 \%$ to $1 \%$ of the population at autopsy. Extraadrenal myelolipomas (EM) are extremely rare with fewer than 50 cases reported. We describe the first case of bilateral EM of the renal sinus. They are difficult to diagnose on imaging alone when arising in this location and biopsies may not yield a definitive answer. Management options include both conservative and surgical approaches depending upon the certainty of the diagnosis, progression of the patient's symptoms and evidence of growth.
\end{abstract}

Can Urol Assoc J 2010;4(6):E164-E168

\section{Case report}

A 62-year-old female presented with a history of vague abdominal pain. Past medical history included insulin dependent type II diabetes, hypertension, hyperlipidemia and hypothyroidism. A total abdominal hysterectomy and salpingo-oophorectomy had been performed 12 years earlier for benign disease. Abdominal ultrasound revealed leftsided hydronephrosis. She underwent cystoscopy and a left retrograde pyelogram, which confirmed a dilated, tortuous left ureter with no stone or obvious cause of obstruction. A non-contrast computer tomography (CT) scan of the abdomen and pelvis showed multiple, bilateral lesions arising from the renal sinuses. The largest was a $10 \times 10 \times 6.9 \mathrm{~cm}$ lobulated mass between the aorta and left kidney (Fig. 1). A magnetic resonance imaging (MRI) scan demonstrated 3 masses in the left renal sinus; a 10.2-cm lesion inferiorly, and 4.2- and 2.2-cm lesions superiorly. Renal lipomatosis was noted. Two identical appearing masses arose from the right renal sinus $(1.9$ and $2.5 \mathrm{~cm})$. Adrenal glands were normal and no lymphadenopathy was detected (Fig. 2). Noncontrast studies were performed due to a transient elevation in creatinine $(209 \mu \mathrm{mol} / \mathrm{L})$.

A CT-guided fine needle aspiration of the left renal sinus mass showed adipose tissue, hemosiderin pigment and hematopoietic tissue, raising the differential diagnosis of extramedullary hematopoiesis $(\mathrm{EH})$ and extra-adrenal myelolipoma (EM). The rare occurrence of both of these entities at this site prompted a repeat core biopsy which demonstrated similar findings but with predominantly adipose tissue and focal aggregates of hematopoietic elements (Fig. 3). Bone marrow assessment was performed to rule out a primary hematopoietic process; findings included slightly hypercellular marrow but with no evidence of a myeloproliferative neoplasm. Molecular studies were negative for BCR-ABL gene transcripts (associated with chronic myelogenous) and for the variant JAK2 allele (associated with polycythemia vera, essential thrombocythemia and idiopathic myelofibrosis).

Active surveillance was initiated, but the patient became increasingly uncomfortable and the larger left-sided renal sinus mass grew. An uncomplicated excision of the left retroperitoneal tumour and kidney was performed.

The surgical specimen including the left kidney with attached adrenal gland and perinephric tissues weighed 1634 g (kidney proper $14.5 \times 6.5 \times 10.0 \mathrm{~cm}$ ). Centered within the renal sinus was a large, lobulated, predominantly pale-yellow 'fatty' mass $(16.0 \times 11.5 \times 10.0 \mathrm{~cm})$ containing irregular islands of red-brown soft tissue (Fig. 4). The mass extended into the perinephric tissues but did not involve the left adrenal gland. It obscured the renal medulla and compressed the renal pelvis with associated dilation of the calyces. Small, sand-like kidney stones were present in the dilated collecting system. Renal hilar vessels were uninvolved. The remaining kidney showed small cysts and slight fetal lobulation of the cortex.

Histological examination revealed a tumour composed of mature adipose and hematopoietic tissues, consistent with myelolipoma (Fig. 5). Hematopoietic tissue included megakaryocytes, maturing granulocytes, lymphocytes and erythroid precursors (Fig. 6). Patchy tumoural hemorrhage and hemosiderin deposition were noted. The kidney demonstrated evidence of arterial and arteriolonephrosclerosis and simple cysts. The uninvolved adrenal showed mild nodular 


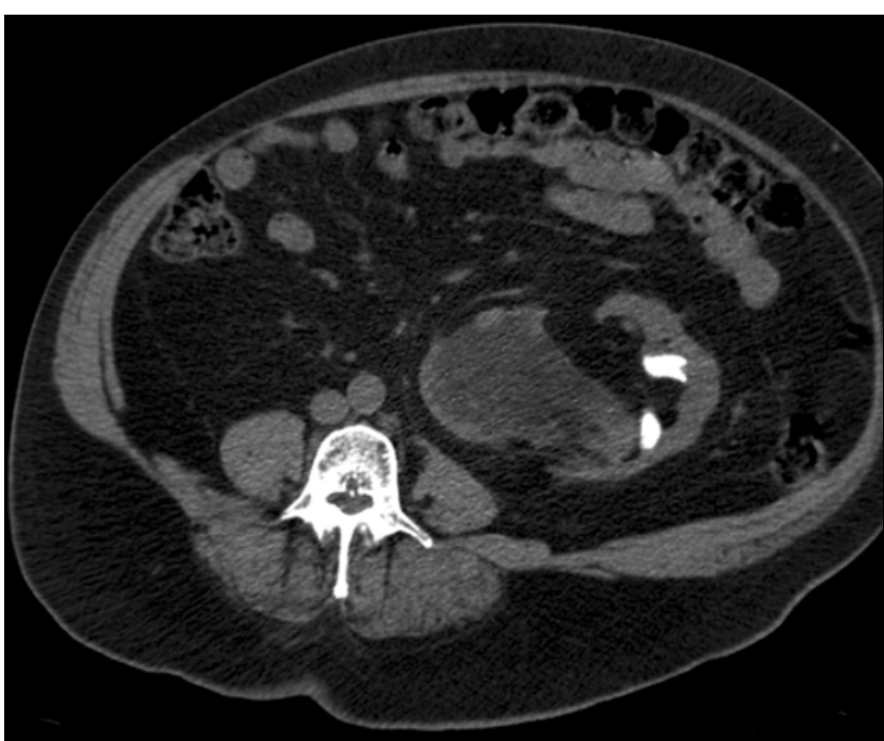

Fig. 1. A computed tomography scan of the abdomen and pelvis.

cortical hyperplasia.

\section{Discussion}

Adrenal myelolipomas are uncommon, benign neoplasms consisting of hematopoietic cellular elements and adipose tissue, found in $0.4 \%$ to $1 \%$ of the population at autopsy. ${ }^{1,2}$ Extra-adrenal myelolipoma is extremely rare with fewer than 50 cases reported. They have been detected in the presacral, pararenal and perirenal retroperitoneum, as well as the mediastinum, thorax, liver and stomach. ${ }^{3}$ Pathogenesis is unclear but theories include development of embryonic mesenchymal remnants, metaplasia of the reticuloendothelial system and emboli of active bone marrow. ${ }^{4}$

As patients are generally asymptomatic, EM is often an incidental finding on ultrasound, CT or MRI. As these tumours are rare, criteria for diagnosing EM radiologically do not exist. Characteristics of adrenal myelolipomas are extrapolated to apply to these structures which present in a variety of locations. ${ }^{5}$ Imaging characteristics of these lesions suggest a benign growth pattern. They are usually well-circumscribed with intact fascial planes. Adipose tissue is characterized by low attenuation on CT imaging (i.e., -25 to -100 Hounsfield units). On MRI, fat displays high signal intensity on T1-weighted images whereas the myeloid component of these tumours has low signal intensity. Contrast enhancement with CT scan or MRI will vary depending on the composition of the mass. Soft tissue components enhance while adipose tissue does not. ${ }^{3}$ Radiologically, it may be difficult to differentiate retroperitoneal EM from other fat containing retroperitoneal masses, of which liposarcoma is the most common. The origin of EM is difficult to predict radiologically. In a previous case report, imaging suggested a renal origin in a myelolipomas that actually arose from the renal hilum. ${ }^{6}$

Biopsy is often required to confirm the diagnosis. In our case, hematopoietic tissue was detected, raising the differential diagnosis of EH and EM. Extramedullary hematopoiesis (hematopoiesis that occurs outside of the medulla of the bone) is associated with hematological disorders, such as thalassemias, myelofibrosis and other states of ineffective hematopoiesis, and is accompanied by chronic anemia, splenomegaly and marked hyperplasia on bone marrow aspirate. ${ }^{7}$ Hematological evaluation of our patient revealed only a mild anemia.

Extramedullary hematopoiesis leading to a localized extramedullary myeloid tumour can be difficult to differentiate from myelolipoma histologically. The main differentiating factor is that $\mathrm{EH}$ is usually a diffuse process with splenomegaly and hepatomegaly and is accompanied by bone marrow abnormalities. Other differential diagnoses may include extensive pyelonephritis with inflammation

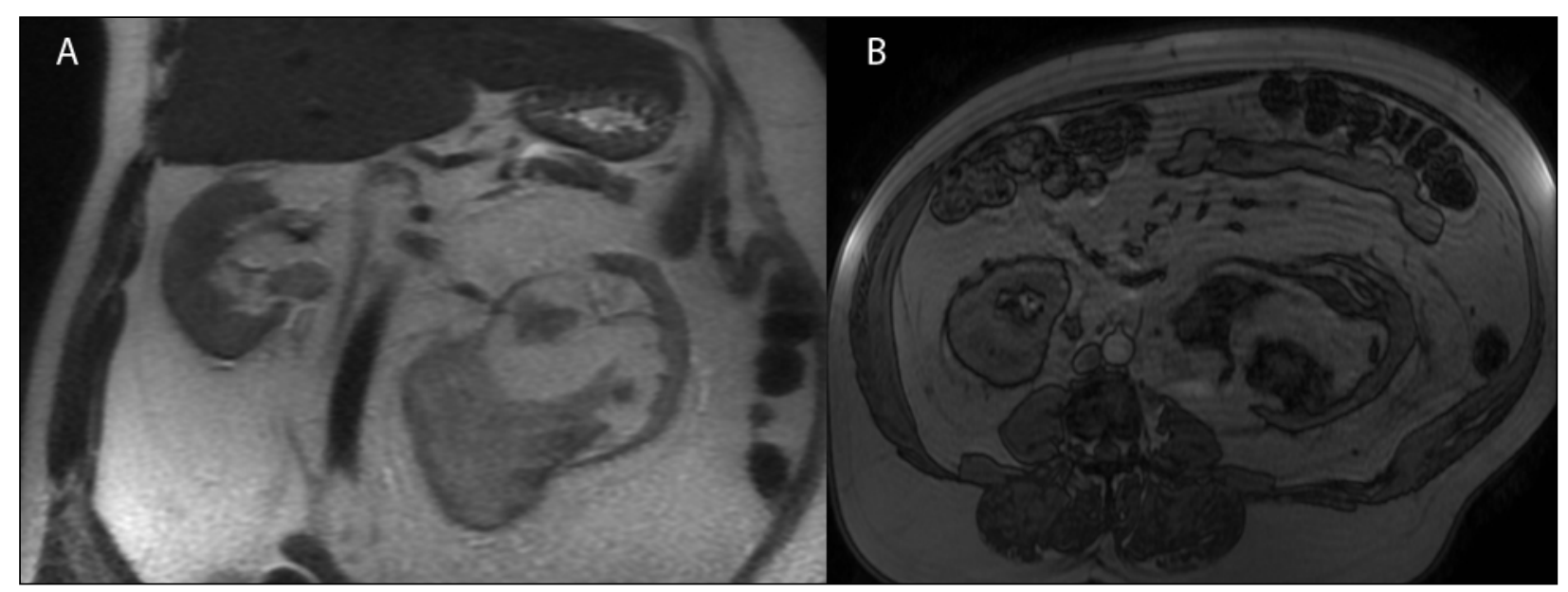

Fig. 2. A magnetic resonance imaging of the abdomen and pelvis. 


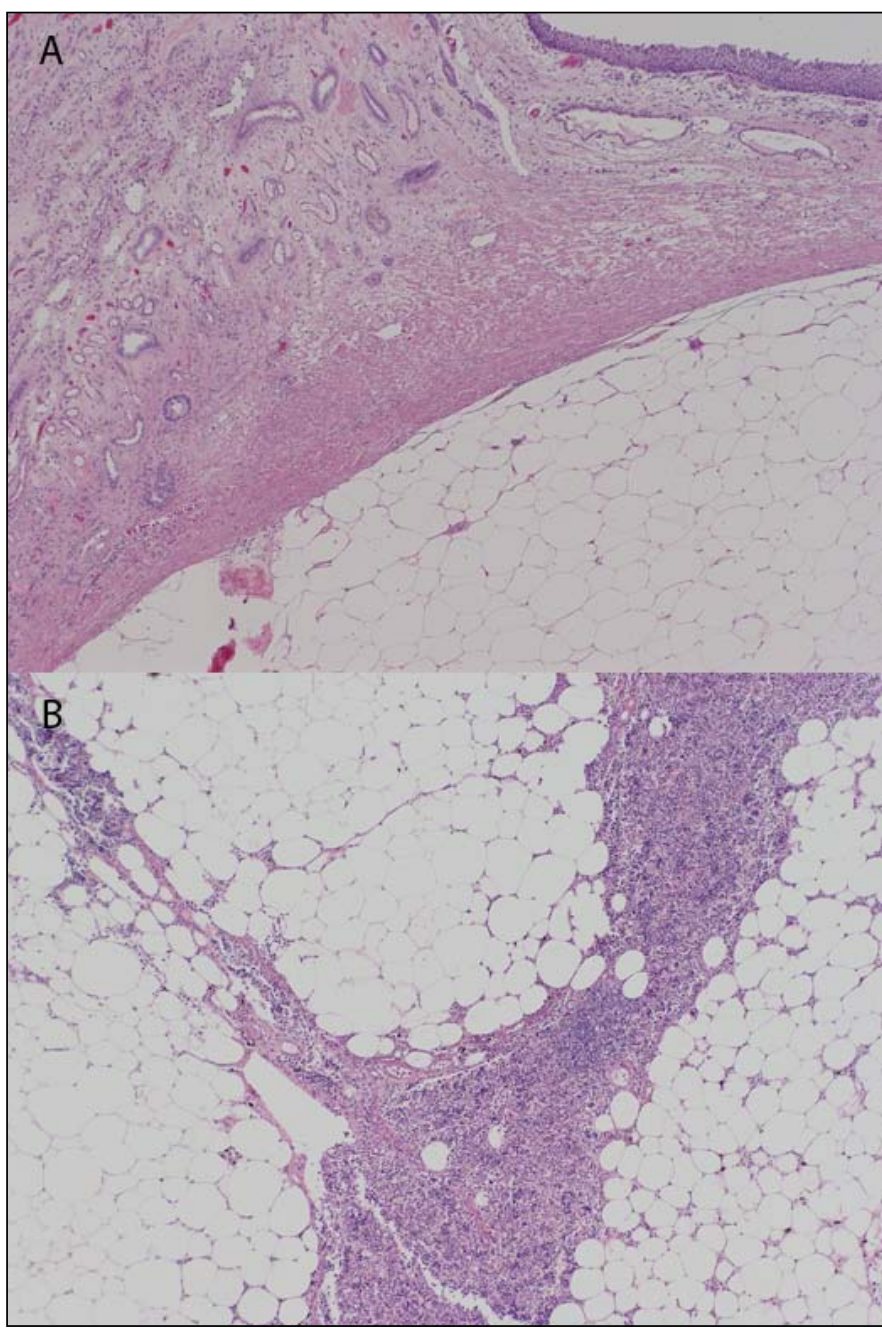

Fig. 3. Hematoxyline and eosin photomicrographs of core biopsy of pararenal mass. (a) Adipose tissue, acute hemorrhage and hemosiderin pigment is seen at left, and hematopoietic tissue, including megakaryocytes, at right (100x). (b) Hematopoietic tissue with maturing myeloid, erythroid, lymphoid and megakaryocytic elements (400x).

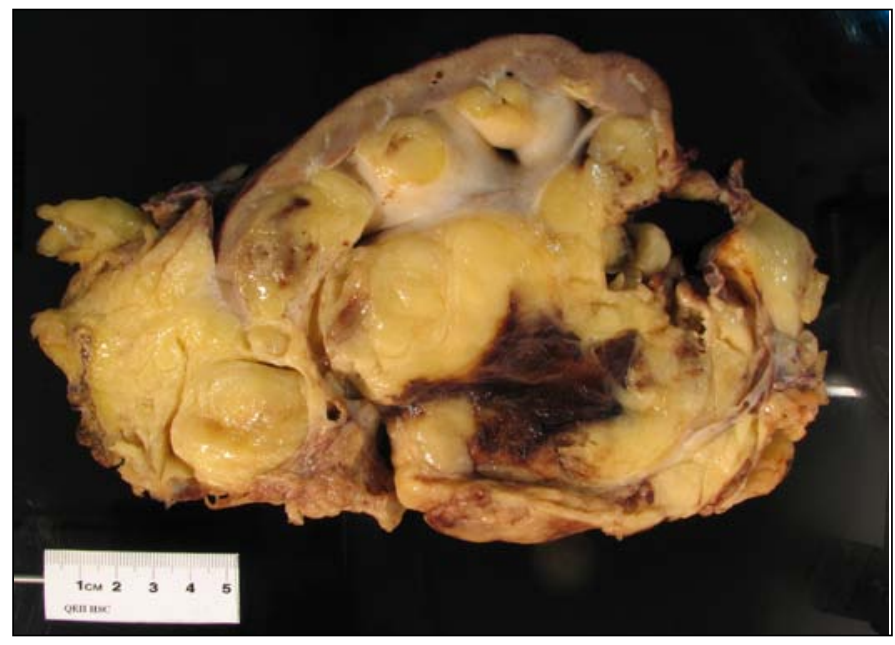

Fig. 4. Coronal slice of resected left kidney and pararenal myelolipoma. An island of hematopoietic tissue appears red-brown and is associated with the adipose tissue. The mass merges with the renal medulla and pelvis with displacement of the proximal ureter (lower right), resulting in dilatation of the collecting system.

extending into the perinephric tissues or other inflammatory masses, but these would lack the megakaryocytes seen in $\mathrm{EM}$ and $\mathrm{EH}$.

Although EMs have been previously described in the renal sinus, ${ }^{8}$ this is the first reported case of bilateral renal sinus EM detected by MRI. Bilateral perirenal EM have been diagnosed in a 77-year-old man who presented with renal failure. The EM encased each kidney within Gerota's fascia causing compression of renal parenchyma. Core biopsy revealed a diagnosis of EM and he was managed non-operatively. The EM in the perirenal space, retroperitoneum or renal sinus may affect the kidney in many ways including causing local symptoms, such as abdominal pain (mass effect), obstructing the urinary tract (hydronephrosis), urinary tract infection and hematuria. Systemic manifestations, such as

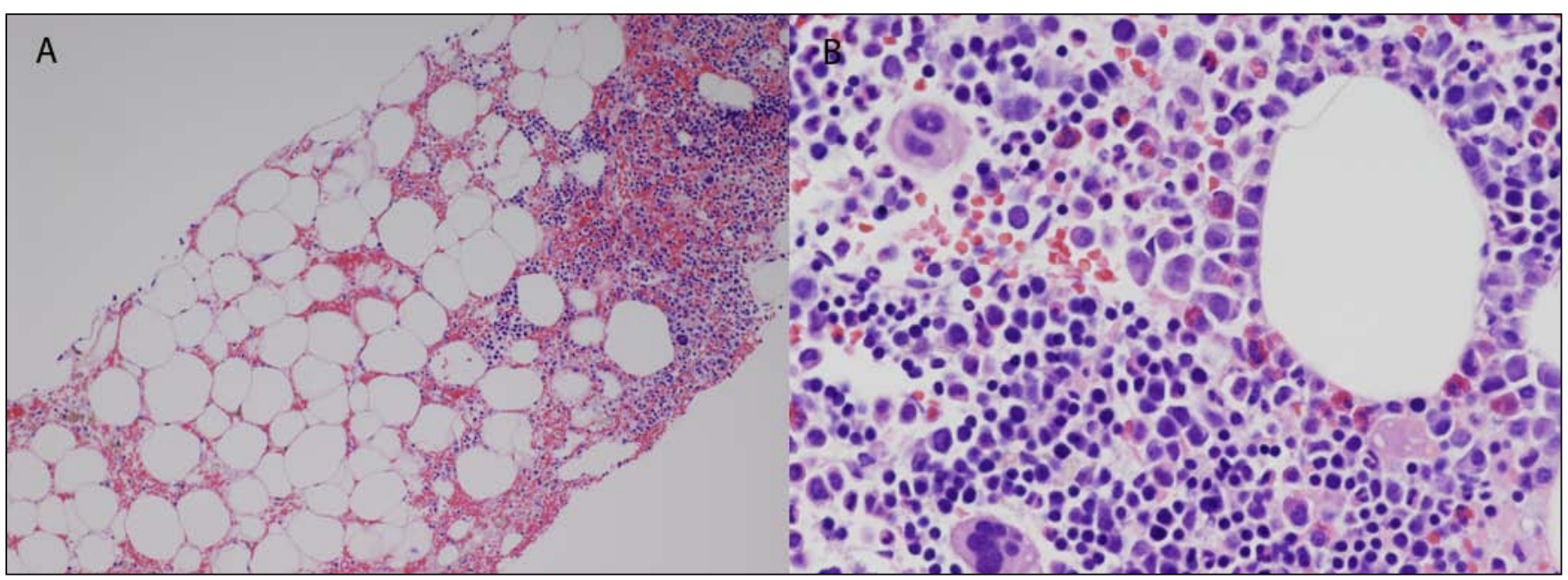

Fig. 5. Hematoxyline and eosin photomicrographs of the nephrectomy specimen (40x). (a) The relationship between the mass (lower right) and the renal medulla (left) and renal pelvis (urothelial lining, upper right) is demonstrated. (b) Hematopoietic elements are seen interspersed within adipose tissue. 


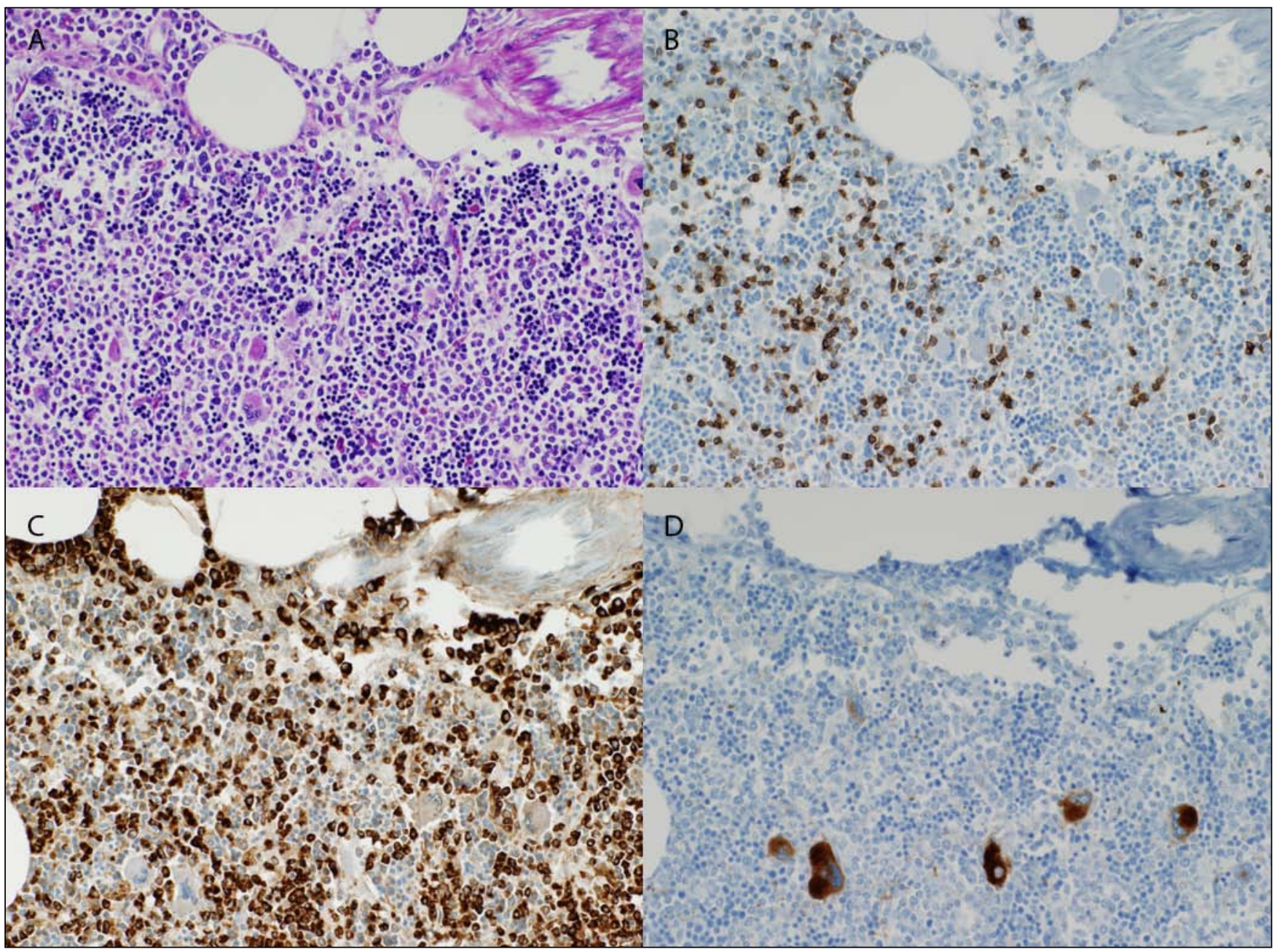

Fig. 6. Photomicrographs of maturing hematopoietic elements within pararenal mass (200x). A periodic acid-Schiff (PAS) stain (a) is used for comparison against a panel of immunohistochemical stains, including leukocyte common antigen (b), myeloperoxidase (c) and CD61 (d), lymphoid, myeloid and megakaryocytic elements, respectively.

weight loss, malaise and weakness, have also been noted. In our case, the tumours arose from the renal sinuses, based on MRI and histological evaluation, and encased the left ureter causing its displacement and obstruction. The MRI showed identical lesions on the right side. It is possible that the MRI appearance of multiple lesions arising from the left renal sinus was the result of nodules of myeloid components within lower attenuation adipose areas and the lobulated contour of the mass.

The usual natural history of EM is benign although they may enlarge and bleed. Some reports have shown stable lesions with follow-up from 3 to 62 months. ${ }^{5,8}$ In patients who are symptomatic, have an unclear diagnosis or possess an enlarging tumour mass, as in our patient, surgical intervention is warranted. It is unknown as to whether the right renal sinus lesions will progress; close radiological follow-up is in progress.

\section{Conclusion}

Management options for EM include conservative and surgical approaches depending upon the certainty of the diagnosis and the progression of the patient's symptoms. Radiological follow-up is warranted due to the potential for growth and hemorrhage.

*Department of Urology, Dalhousie University, Halifax, NS; 'Division of Anatomical Pathology and Department of Pathology, Dalhousie University, Halifax, NS; §Division of Hematology, Dalhousie University, Halifax, NS 
Cox et al.

Competing interests: None declared.

This paper has been peer-reviewed.

\section{References}

1. Olsson CA, Krane RJ, Klugo RC, et al. Adrenal myelolipoma. Surgery 1973;73:665-70.

2. Asuncion CM, Loh WP. Myelolipoma of the adrenal gland. J Indiana State Med Assoc 1965;58:38-43.

3. Kammen B, Elder DE, Fraker DL, et al. Extraadrenal myelolipoma: MR imaging findings. AJR Am J Roentgenol 1998;171:721-3.
4. Nguyen B. Retroperitoneal extraadrenal myelolipoma: Technitium-99m sulfur colloid scintigraphy and CT imaging. Clin Nucl Med 2007;32:135-8.

5. Kumar M, Duerinckx A. Bilateral extraadrenal perirenal myelolipomas: An imaging challenge. AJR Am J Roentgenol 2004; 183:833-6.

6. Wagner JR, Kleiner DE, Walther MM, et al. Perirenal myelolipoma. Urology 1997;49:128-30.

7. Talwalker $S$, Shaheen $S$. Extra-adrenal myelolipoma in the renal hilum: A case report and review of the literature. Arch Pathol Lab Med 2006;130:1049-52.

8. Amin MB, Tickoo SK, Schultz D. Myelolipoma of the renal sinus: An unusual site for a rare extra-adrenal lesion. Arch Pathol Lab Med 1999:123:631-4.

Correspondence: Dr. Ashley Cox, Department of Urology, Room 294-5S, VG-5th Floor, Centennial Building, QEll Health Science Centre, 1278 Tower Rd, Halifax, NS B3H 2Y9; ashleycox@dal.ca 